03

\title{
Колебания неосесимметричного цилиндра в заполненной жидкостью полости, совершающей вращательные осцилляции
}

\author{
() В.Д. Щипицын
}

Лаборатория вибрационной гидромеханики, Пермский государственный гуманитарно-педагогический университет, Пермь, Россия

E-mail: schipitsyn@pspu.ru

Поступило в Редакцию 21 апреля 2020г.

В окончательной редакции 6 мая 2020г.

Принято к публикации 6 мая 2020г.

Экспериментально исследовано поведение цилиндрического тела неосесимметричного сечения в горизонтально расположенной цилиндрической кювете, заполненной вязкой несжимаемой жидкостью, при вращательных колебаниях. Обнаружен и изучен эффект вибрационного подвеса тяжелого неосесимметричного цилиндра вблизи дна вибрирующей полости на расстоянии, сравнимом с толщиной вязкого слоя Стокса. Исследованы характер осцилляционной динамики тела и его взаимодействие с границей полости посредством скоростной видеорегистрации процесса.

Ключевые слова: подъемная сила, неосесимметричное цилиндрическое тело, вязкая жидкость, вращательные вибрации, гидродинамическое взаимодействие.

DOI: 10.21883/PJTF.2020.15.49749.18349

Экспериментальное исследование вибрационной подъемной силы, действующей на круговой цилиндр в жидкости вблизи границы цилиндрической полости, совершающей высокочастотные вращательные вибрации, было представлено в работах $[1,2]$. В случае поступательных вибраций сила может быть настолько интенсивной, что обеспечивает подвес тяжелого твердого тела вблизи потолка полости (или удерживает легкое тело на дне) в гравитационном поле [3]. Изучению подъемной силы, действующей на тело неосесимметричной цилиндрической формы в прямоугольной полости, заполненной жидкостью и совершающей поступательные горизонтальные вибрации, посвящена работа [4]. Динамика тяжелого кругового цилиндра практически нейтральной плавучести в цилиндрической полости, заполненной жидкостью и подверженной модулированному вращению, была экспериментально исследована в [5]. Описание механизма генерации подъемной силы при колебаниях малой амплитуды симметричного тела в невязкой жидкости дано еще в [6]. Осредненное действие осциллирующих потоков на твердые тела изучалось в [7]. Основное внимание было уделено крупномасштабным колебаниям большой амплитуды. Теоретические исследования сил, действующих на осциллирующие в жидкости симметричные тела, в неакустическом приближении проводились в [8]. В экспериментальной работе [9] показано, что подъемная сила возникает и в случае, когда тангенциальные колебания тела нормальны к границе полости. Численное 3D-моделирование подъемной силы, действующей на взвешенные сферические частицы в контейнере, заполненном жидкостью и осциллирующем в горизонтальном направлении, выполнено в [10].
Данная публикация является логическим продолжением работ [1-5] и находится в хорошем согласии с результатами экспериментов, описанных в них. Основная особенность работы состоит в том, что опыты проводились с неосесимметричным телом. Решаемая задача о вибрационном управлении твердыми неосесимметричными включениями в вязкой жидкости имеет большой прикладной потенциал. Обнаруженные эффекты могут послужить предпосылкой для разработки эффективных технологий очистки жидкостей от примесей и создания прототипа так называемого вибрационного фильтра очистки жидкостей. Изучение динамики твердых включений асимметричной формы особенно актуально, поскольку такая постановка задачи максимально приближена к моделированию поведения мусора в очищаемой жидкости.

В ходе экспериментов тяжелый цилиндр эллиптического сечения 1 помещается в горизонтально расположенную цилиндрическую оргстеклянную кювету 2, полностью заполненную вязкой несжимаемой жидкостью, и закрепляется на столике электродинамического вибратора 3 (рис. 1,a). Кювета может совершать вращательные колебания вокруг горизонтальной оси симметрии по гармоническому закону $\varphi=\varphi_{0} \cos (\Omega t)$. Амплитуда угловых колебаний полости $\varphi_{0}$ и линейная частота вибрационного воздействия $f \equiv \Omega / 2 \pi$ в ходе экспериментов варьируются в интервалах $\varphi_{0}=0.001-0.2 \mathrm{rad}$, $f=0.1-50 \mathrm{~Hz}$. Кювета представляет собой прозрачную плексигласовую трубу с внутренним радиусом $R=65.0 \mathrm{~mm}$ и длиной $250 \mathrm{~mm}$. В качестве рабочей жидкости используется водоглицериновый раствор (кинематическая вязкость $v=3.9 \mathrm{St}$, плотность жидкости $\left.\rho_{L}=1.25 \mathrm{~g} / \mathrm{cm}^{3}\right)$. Основные эксперименты выполняются с неосесимметричным цилиндром (длина цилиндра 

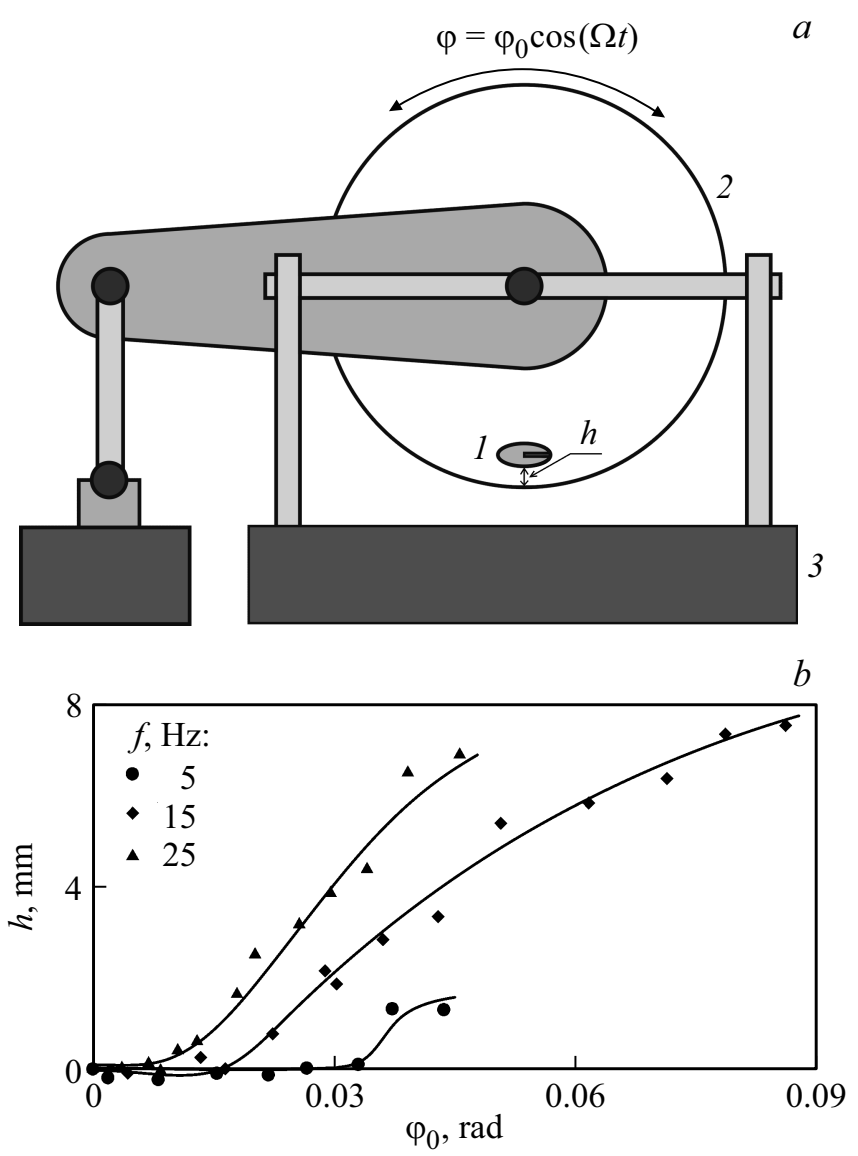

Рис. 1. $a-$ схема экспериментальной установки (пояснения в тексте), $b-$ зависимость расстояния $h$ от амплитуды колебаний полости для различных частот вибраций.

$l=242 \mathrm{~mm}$, большой диаметр $d_{a}=8.95 \mathrm{~mm}$, малый диаметр $d_{b}=6.31 \mathrm{~mm}$, средняя плотность $\rho_{S}=1.38 \mathrm{~g} / \mathrm{cm}^{3}$, относительная плотность $\left.\rho \equiv \rho_{S} / \rho_{L}=1.11\right)$. Как было показано в предыдущих работах нашего авторского коллектива [1-5], характерная относительная длина цилиндра не является управляющим параметром в эффекте генерации вибрационной подъемной силы. Основная часть визуальных наблюдений за поведением тела проводится через прозрачный торец горизонтально расположенной цилиндрической кюветы. Для того чтобы исключить возможность смещения цилиндра в глубь кюветы в ходе эксперимента и таким образом сохранить фокусировку камеры на переднем торце цилиндра при фото- и видеосъемке эксперимента, изготавливается тело с длиной, практически равной длине полости.

Обнаружено, что вращательные колебания полости приводят к генерации вибрационной подъемной силы, обеспечивающей квазистационарный подвес тяжелого неосесимметричного цилиндра на некотором расстоянии $h$ от дна полости в поле силы тяжести. На рис. $1, b$ показано изменение величины зазора $h$ с амплитудой угловых колебаний полости $\varphi_{0}$ для различных фиксированных значений частоты вибраций $f$. Для всех серий экспериментов, представленных на этом рисунке, характерно однотипное поведение: пороговое отталкивание тела и его монотонное удаление с повышением амплитуды вибраций; возвращение тела ко дну полости при понижении амплитуды вибраций. Обнаружено, что с повышением частоты вибраций $f$ амплитуда пороговых переходов тела смещается в область меньших значений $\varphi_{0}$.

Колебательная динамика тяжелого неосесимметричного цилиндра изучается при помощи высокоскоростной видеорегистрации процесса. При вибрациях малой интенсивности под действием силы тяжести тяжелое тело находится на дне кюветы. По достижении критического значения угловой амплитуды полости $\varphi_{0}$ (частота $f$ фиксирована) тело пороговым образом отрывается от нижней границы полости (рис. 2). Вращательные колебания полости, происходящие в лабораторной системе по гармоническому закону $\varphi=\varphi_{0} \cos (\Omega t)$ (рис. 2, $a$, кривая 1), вызывают тангенциальные колебания тела в системе отсчета полости по закону $\beta=\beta_{0} \cos (\Omega t+\gamma)$, где $\beta_{0}$ - амплитуда колебаний, $\gamma$ - сдвиг фаз (кривая 2). Можно заметить, что колебания тела и полости происходят практически в противофазе $(\gamma \approx 180 \mathrm{deg})$, амплитуда колебаний тела $\beta_{0}$ немного меньше амплитуды колебаний полости $\varphi_{0}$. В процессе колебаний тело ориентируется своей большей осью поперечного сечения параллельно границе полости (так, как это изображено на рис. $1, a)$; его угловая координата $\alpha \approx 90 \mathrm{deg}$. Траектория осциллирующего движения тела относительно полости представляет собой практически прямую
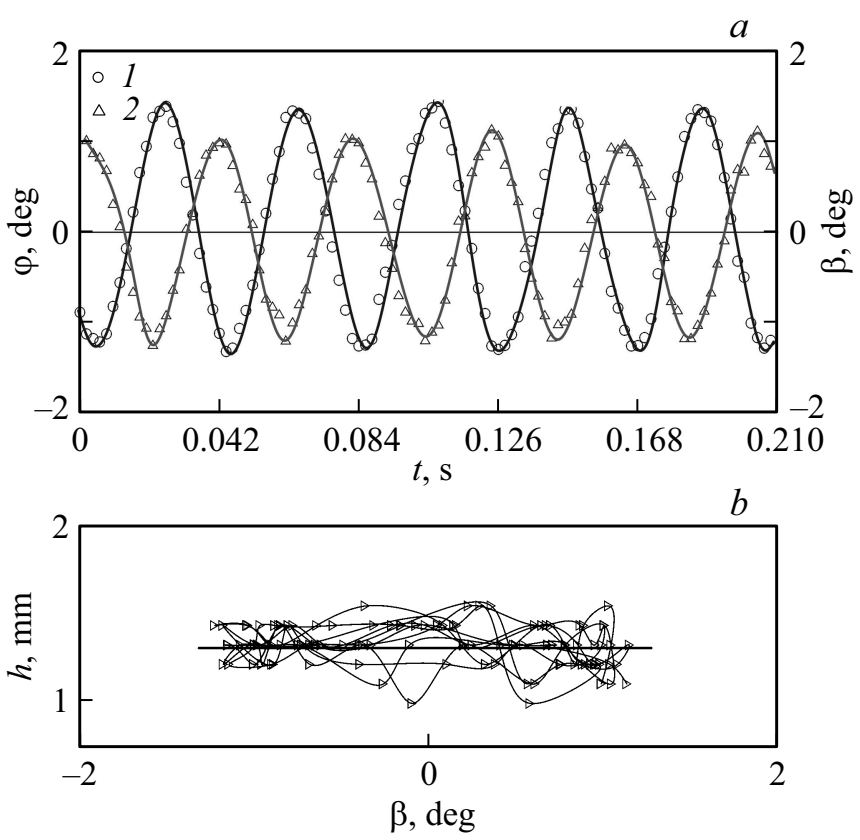

Рис. 2. $a-$ изменение со временем угловой координаты полости $\varphi$ в лабораторной системе отсчета $(1)$ и координаты тела $\beta$ в системе отсчета полости (2), $b$ - траектория колебаний тела после отрыва от дна полости; $f=25 \mathrm{~Hz}$, $\varphi_{0}=0.02 \mathrm{rad}$. 

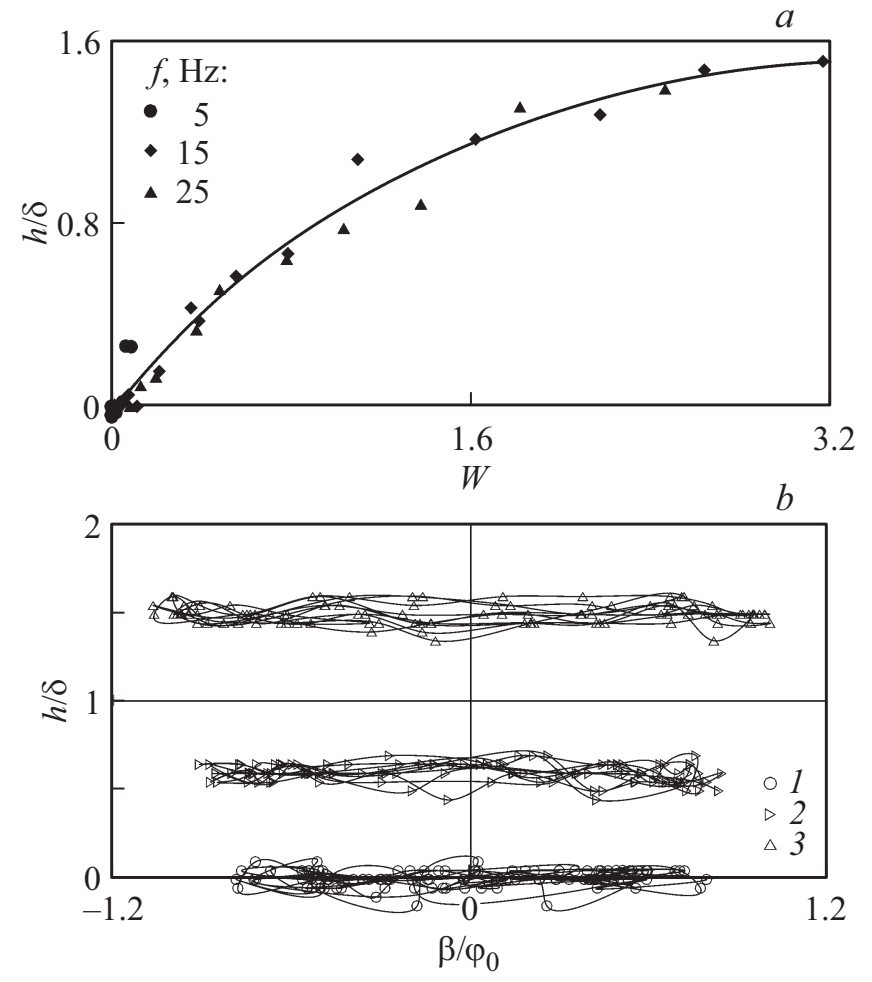

Рис. 3. Зависимость безразмерного расстояния $h / \delta$ между телом и нижней границей полости от безразмерного параметра $W(a)$ и траектории осциллирующего движения тела относительно полости (b) до отрыва $(1)$, после отрыва (2) и при большей амплитуде надкритичности (3).

(рис. $2, b)$. Величина зазора между телом и дном полости в течение периода практически не меняется: $h \approx 1.2 \mathrm{~mm}$. Небольшое расхождение в величине зазора на рисунке связано с погрешностью, вызванной дискретностью шага измерительной системы.

В работе [11] было показано, что определяющими динамику цилиндра кругового сечения в поле силы тяжести являются безразмерный вибрационный параметр $W$ и безразмерная частота вибраций $\omega$. С учетом особой геометрии тела и типа вибрационного воздействия безразмерные комплексы, введенные ранее в [11], принимают вид $W=\left(\varphi_{0} R\right)^{2} \Omega^{2} / g d_{a}, \omega=\Omega d_{a}^{2} / v$.

Обнаружено, что все результаты по отталкиванию тяжелого цилиндра от дна полости, полученные при изменении значений $f$, на плоскости управляющих параметров $W, h / \delta$ удовлетворительно группируются вблизи одной кривой, вязкость жидкости в ходе экспериментов не менялась (рис. 3,a). Квазиравновесие тяжелого тела вблизи дна полости возможно на расстоянии, сравнимом с толщиной слоя Стокса, характеризующего область существования вибрационной силы отталкивания $(h / \delta \approx 2)$.

Проанализируем результаты, полученные с помощью скоростной видеорегистрации процесса. Проведем обезразмеривание расстояния между телом и стенкой кюветы через толщину слоя Стокса $\delta$, угловое смещение тела $\beta$ - через амплитуду угловых колебаний полости $\varphi_{0}$. В том случае, когда тяжелый эллиптический цилиндр находится вблизи нижней границы полости $(\omega=32$, $W=0.12)$, он совершает тангенциальные колебания с относительной амплитудой $\beta / \varphi_{0} \approx 0.7$, расстояние между телом и нижней стенкой кюветы отсутствует (рис. 3, b, кривая 1). Безразмерная траектория колебаний тела в надкритическом режиме (после его отрыва от дна полости) представлена кривой $2(\omega=32$, $W=0.47)$. Между телом и полостью появляется зазор $(h / \delta \approx 0.6)$, амплитуда колебаний немного увеличивается $\left(\beta / \varphi_{0} \approx 0.8\right)$. Дальнейшее повышение надкритичности (кривая $3 ; \omega=32, W=1.92$ ) приводит к еще большему удалению цилиндра от нижней стенки $(h / \delta \approx 1.5)$. Амплитуда тангенциальных колебаний тела относительно кюветы по сравнению с предыдущим случаем также немного увеличивается $\left(\beta / \varphi_{0} \approx 1\right)$.

Таким образом, в работе экспериментально обнаружено, что вращательные колебания полости с жидкостью приводят к возникновению вибрационной подъемной силы, способной оттолкнуть тяжелое неосесимметричное цилиндрическое тело от дна кюветы и удерживать его в состоянии квазистационарного подвеса в поле силы тяжести на расстоянии, сравнимом с толщиной слоя Стокса. Обнаруженное явление имеет потенциал применения для контроля и разделения многофазных систем.

\section{Финансирование работы}

Работа выполнена при финансовой поддержке Российского научного фонда (проект № 18-71-00122).

\section{Конфликт интересов}

Автор заявляет, что у него нет конфликта интересов.

\section{Список литературы}

[1] Ivanova A.A., Kozlov V.G. // Microgravity Sci. Technol. 2014. V. 26. N 3. P. 179-187. https://doi.org/10.1007/s12217-014-9394-9

[2] Ivanova A., Kozlov V., Schipitsyn V., Stambouli M., Stambouli $M$. Lift force acting on solid in liquid near the boundary performing tangential oscillations // Proc. of the 64th International Astronautical Congress (IAC-2013). Beijing, China, 2013. P. IAC-13-A2.P7 (1-8).

[3] Иванова А.А., Козлов В.Г., Кузаев А.Ф. // Изв. РАН. Механика жидкости и газа. 2008. Т. 43. № 2. С. 31-40. [Пер. версия: https://doi.org/10.1134/s001546280802004x]

[4] Schipitsyn V., Kozlov V. Quasistationary states of a nonaxisymmetric cylindrical solid under translational vibrations in liquid // Abstr. 8th Int. Symp. on bifurcations and instability in fluid dynamics (BIFD-2019). Limerick, Ireland, 2019. P. 278.

[5] Власова О.А., Козлов В.Г., Козлов Н.В. // ПМТФ. 2018. T. 59. № 2. C. 39-49. DOI: 10.15372/PMTF20180205 [Пер. версия: https://doi.org/10.1134/s0021894418020050].

[6] Ламб Г. Гидродинамика. М.-Л.: ОГИЗ, 1947. 928 с. 
[7] Sumer M., Fredsoe J. Hydrodynamics around cylindrical structures. Singapore-London: World Scientific Publ., 2006. $530 \mathrm{p}$.

[8] Луговиов Б.А., Сенницкий В.Л. // ДАН. 1986. Т. 289. № 2. C. 314-317. http://mi.mathnet.ru/eng/dan47587

[9] Hassan S., Lyubimova T.P., Lyubimov D.V., Kawaji M. // Int. J. Multiphase Flow. 2006. V. 32. N 9. P. 1037-1054. https://doi.org/10.1016/j.ijmultiphaseflow.2006.05.008

[10] Saadatmand M., Kawaji M., Hu H. // Microgravity Sci. Technol. 2012. V. 24. N 1. P. 53-64. https://doi.org/10.1007/s12217-011-9291-4

[11] Kozlov V.G. // Europhys. Lett. 1996. V. 36. N 9. P. 651-656. https://doi.org/10.1209/epl/11996-00282-0 